\title{
National trends in beverage consumption in children from birth to 5 years: analysis of NHANES across three decades
}

Victor L Fulgoni $\| I^{1 *}$ and Erin E Quann ${ }^{2}$

\begin{abstract}
Background: Given the epidemic of childhood obesity, it is crucial to assess food and beverage intake trends. Beverages can provide a large number of calories and since consumption patterns seem to develop at a young age we examined beverage consumption trends over three decades. The objective of this study was to assess the beverage (milk, fruit juice, fruit drinks, tea, soy beverages, and soft drinks) consumption trends in children $<1-5$ years of age.

Methods: Data from individuals ages $<1-5$ years participating in the National Health and Nutrition Examination Survey (NHANES) from 1976-1980, 1988-1994 and 2001-2006 were used to assess beverage consumption and associated calorie and nutrient intakes.

Results: During the NHANES 1976-1980 and 1988-1994 periods, approximately 84-85\% of children were consuming milk, whereas only 77\% were consuming milk during NHANES 2001-2006. Flavored milk intake was relatively low, but increased to $14 \%$ during the last decade $(p<0.001)$. Fruit juice consumption increased dramatically during NHANES 2001-2006 to more than 50\% of the population compared to about 30\% in the older surveys $(p<0.001)$. No significant changes were observed in fruit drink intake across all three decades with $35-37 \%$ of this population consuming fruit drinks. At least 30\% of children consumed soft drinks. Milk was the largest beverage calorie contributor in all three decades surveyed and was the primary contributor of calcium (52-62\%), phosphorus (37-42\%), magnesium (27-28\%), and potassium (32-37\%). Fruit juice and fruit drinks each provided 8-10\% of calories with soft drinks providing 5-6\% of calories. Fruit juice was an important provider of potassium (16-19\%) and magnesium (11\%). Fruit drinks provided less than $5 \%$ of nutrients examined and soft drinks provided very little of the nutrients evaluated.
\end{abstract}

Conclusions: Given concerns about childhood obesity and the need to meet nutrition requirements, it is prudent that parents, educators and child caretakers replace some of the nutrient poor beverages young children are currently consuming with more nutrient dense sources like low-fat and fat-free milk.

Keywords: NHANES, Pre-school children, Beverages, Milk, Fruit juice, Fruit drinks, Soft drinks, Nutrient intakes

\footnotetext{
* Correspondence: vic3rd@aol.com

'Nutrition Impact, LLC, 9725 D Drive North, Battle Creek, MI 49104, USA

Full list of author information is available at the end of the article
} 


\section{Introduction}

A child's first 5 years of life is characterized by rapid growth and development and is the period during which food preferences and behaviors develop that often serve as the foundational basis for future eating habits $[1,2]$. Beverage consumption patterns developed during the early years can have lasting implications throughout childhood and adolescence and into adult years. The American Academy of Pediatrics (AAP) provides recommendations for the introduction of beverages to children's diets. They discourage fruit juice intake before the infant is at least 4-6 months of age and fruit drinks, sports drinks and energy drinks are generally discouraged all together [3-5]. The introduction of cow's milk is recommended after 1 year of age and low-fat and fat-free varieties are encouraged once children turn 2 years old [3]. The 2010 Dietary Guidelines for Americans acknowledge the important link between childhood and adulthood beverage consumption by reinforcing the important habit of drinking milk during childhood because "those who consume milk at an early age are more likely to do so as adults" [6].

In the nationwide sample of infants and toddlers aged 4-24 months who participated in the Feeding Infants and Toddlers Study, beverages provided 36\% of the total daily energy [7]. According to 2005-2006 National Health and Nutrition Examination Survey (NHANES) data, total beverage intake among children ages $2-6$ years accounts for an average of $367 \mathrm{kcal} /$ day [8]. Although milk intake accounted for the greatest amount of fluids consumed in this young age group ( $331 \mathrm{ml} /$ day, on average), sodas and fruit drinks provided $206 \mathrm{ml}$ of average daily fluids and by the time children were 7 years of age and older, sodas accounted for the most fluid intake, aside from water, while milk intake continued to decline [8]. The type of beverages consumed can have a significant impact on diet quality and nutrient adequacy.

Lower milk intake makes it more difficult for young children to meet daily milk and milk product group and nutrient intake recommendations, and it could have negative impacts on development since milk and the nutrients it provides are associated with growth and bone health in preschool children $[9,10]$. Health professionals are concerned that high calorie, high sugar, nutrient poor beverages like soft drinks and fruit-flavored drinks displace nutrient rich beverages such as milk [3]. Sugar-sweetened beverage consumption, primarily referring to carbonated beverages and fruit drinks, begins during the preschool years and generally increases with age [11-13]. The consumption of milk by preschoolers has been positively correlated with diet quality and meeting nutrient recommendations, including vitamin A, folate, vitamin B12, calcium, and magnesium, whereas sugar-sweetened beverage consumption, including soda and fruit drinks, by young children has been linked to reduced milk intake and negatively correlated with achieving intakes of vitamin $\mathrm{A}$, vitamin $\mathrm{C}$, and calcium [14-16].

Research indicates a link between beverage consumption and body weight as well. Childhood overweight and obesity has increased at alarming rates in the United States. Even among children aged 2-5 years of age, obesity defined as body mass index (BMI) greater than the 95th percentile for age, increased from 5 to $10.4 \%$ between 1976-1980 and 2007-2008 [17]. There is evidence that a higher intake of sugar-sweetened beverages, such as soda, is associated with increased energy intake [18] and body weight and/or adiposity in children $[6,18]$. Several studies have observed this phenomenon in preschool children $[12,19,20]$.

In light of the importance of beverage consumption to diet quality and obesity among preschoolers, the purpose of this study was to identify beverage trends in young children over the past three decades. Particular emphasis was placed on evaluating the role of milk and its possible displacement with the rising consumption of empty calorie beverages at this early age.

\section{Methods}

Our analysis utilized beverage intake data available from NHANES 1976-1980, 1988-1994, and 2000-2006 for children less than 1 year of age to 5 years of age. NHANES is an ongoing cross-sectional nationally representative health and nutrition examination survey of a stratified, multistage probability sample of the civilian, non-institutionalized U.S. population [21]. Beverage consumption data was based on 24-hour dietary recalls collected in each NHANES cycle in which proxy respondents reported on all foods and beverages consumed by the children in a 24-hour period. While multiple days of recall were obtained in some NHANES surveys, only one day of recall was used for each subject to allow comparison over time. Participants whose records were incomplete, unreliable, or were being breastfed were excluded. Data from NHANES 1976-1980 included only children 6 months and older, whereas the more recent NHANES included children from birth.

Beverage categories were identified as: milk [total as well as white, flavored (sugar-sweetened), whole, $2 \%, 1 \%$, and fat-free separately], $100 \%$ fruit juice, fruit drinks, soft drinks, tea and soy beverages. White milk included all non-flavored fluid milk consumed as beverages as well as milk consumed with other foods such as cereal and macaroni and cheese. Flavored milk was defined as flavored fluid milk purchased as such (i.e., chocolate, strawberry) or milk prepared by the addition of flavored syrups or powder. Fruit drinks were beverages with less than 100\% juice; most contained added sugars. Soft drinks included both "regular" and "diet" sodas. The percentage of the population that consumed each beverage 
category as well as the amounts consumed by those children was determined by age. The mean intake and percent of total intake of calories, total fat, saturated fat, protein, calcium, phosphorus, magnesium (for NHANES 1988-1994 and 2001-2006 only), and potassium were determined for each beverage category using the respective nutrient content information from each NHANES study period. NHANES 1976-1980 did not measure magnesium intake.

All analyses of percentages and/or means with standard errors were conducted using SAS version 9.2/SUDAAN version 10.0 (SAS Institute, Cary, NC/RTI, Research Triangle Park, NC) to adjust for the complex sampling design of each NHANES survey. Pair-wise comparisons between NHANES 1976-1980 and NHANES 1988-1994, 10-14\% NHANES 1988-1994 and NHANES 2001-2006 and NHANES 1976-1980 and NHANES 2001-2006 were performed on mean percents and means using $z$ scores to identify significant differences.

\section{Results}

A total sample of 3,998 children $<1-5$ years of age was utilized from NHANES 1976-1980, 6,871 from NHANES 1988-1994 and 4,430 from NHANES 2001-2006.

\section{Beverage choice (Percentage use)}

Across all three decades, milk was the beverage consumed by most young children (Figure 1). There was a significant decrease $(\mathrm{p}<0.001)$ in milk consumption in the most recent NHANES surveys compared to the previous surveys. During the NHANES 1976-1980 and 1988-1994 periods, approximately $84-85 \%$ of children in this age group were consuming milk, whereas only 77\% were consuming milk during NHANES 2001-2006.
Flavored milk intake was relatively low during NHANES 1976-1980 and NHANES 1988-1994, but increased to $14 \%$ during the last decade $(\mathrm{p}<0.001)$. Fruit juice consumption increased dramatically in this age group during NHANES 2001-2006 to more than 50\% of the population compared to about $30 \%$ in the older surveys $(\mathrm{p}<0.001)$. No significant changes were observed in fruit drink intake across all three decades. On average, 35$37 \%$ of this population consumed fruit drinks. In the case of soft drinks, at least $30 \%$ of children consumed this drink on any given day over the past 30 years. During the NHANES 1988-1994 period, there was a significant increase to $36 \%(\mathrm{p}<0.001)$ which leveled back to $30 \%$ in NHANES 2001-2006. Percent of children consuming tea significantly decreased from 10-14\% from previous decades to 7\% during NHANES 2001-2006. Consumption of soy beverages were not recorded in the earlier surveys and intake was less than $1 \%$ in NHANES 2001-2006. In the remaining analyses, tea and soy beverages were excluded because less than $10 \%$ of young children consumed these beverages.

Age differences in beverage trends across decades were assessed in relation to total milk, flavored milk, fruit juice, fruit drink, and soft drinks consumption (Table 1). Among children less than 1 year old, there was an almost $11 \%$ increase in milk consumption in NHANES 2001-2006 compared to NHANES 1988-1994. From the age of $1-5$ years, the proportion of children that consumed milk was similar for the earlier two decades, whereas during NHANES 2001-2006 there was a tendency for fewer children to consume milk after the age of 3 years. For children $1-3$ and 5 years, there was a significant increase $(\mathrm{p}<0.05)$ in the percent of children who were consuming milk during NHANES 1988-1994

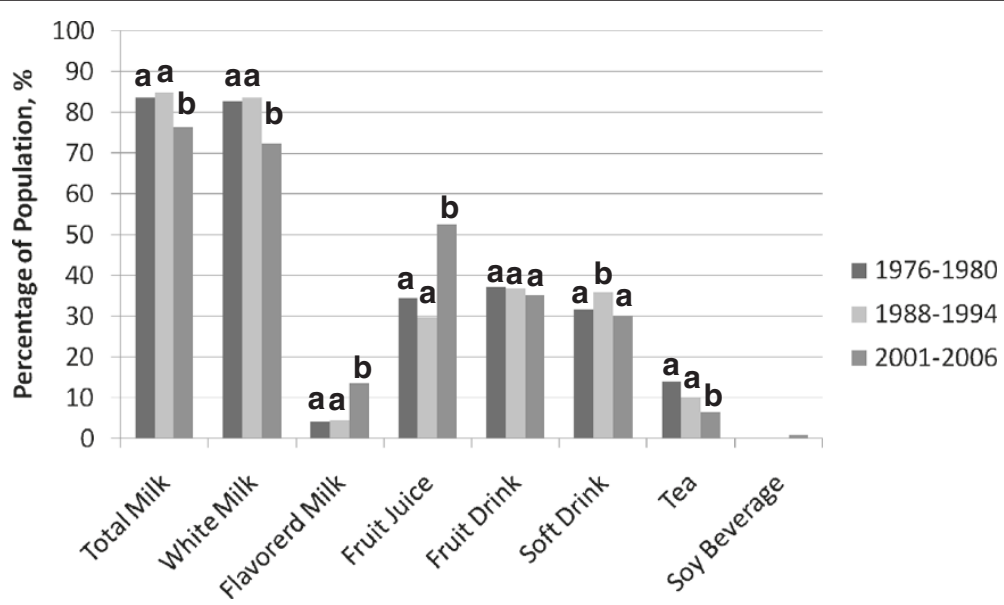

Figure 1 Percentage of children <1-5 years of age consuming different beverages during NHANES 1976-1980, NHANES 1988-1994, and NHANES 2001-2006. NHANES - National Health and Nutrition Examination Survey. Columns with different letters within beverage category are significantly different $(p<0.01)$. 
Table 1 Percent of children <1-5 years that consumed each beverage in NHANES 1976-1980, NHANES 1988-1994, and NHANES 2001-2006, categorized by age

\begin{tabular}{|c|c|c|c|c|c|c|c|c|c|}
\hline Beverage & $\begin{array}{l}\text { NHANES } \\
1976-1980^{*}(\mathrm{~A})\end{array}$ & $\begin{array}{l}\text { NHANES } \\
\text { 1988-1994 (B) }\end{array}$ & $\begin{array}{l}\text { NHANES } \\
2001-2006(C) \\
\end{array}$ & $\begin{array}{l}\text { Change } \\
\text { (A-B) }\end{array}$ & $\begin{array}{l}\text { p-value } \\
\text { (A-B) }\end{array}$ & $\begin{array}{l}\text { Change } \\
\text { (B-C) }\end{array}$ & $\begin{array}{l}\text { p-value } \\
\text { (B-C) }\end{array}$ & $\begin{array}{l}\text { Change } \\
(\mathrm{A}-\mathrm{C})\end{array}$ & $\begin{array}{l}\text { p-value } \\
(\mathrm{A}-\mathrm{C})\end{array}$ \\
\hline \multicolumn{10}{|l|}{ Total Milk } \\
\hline$<1$ year & N/A & $3.1 \pm 0.7^{1}$ & $13.8 \pm 1.3$ & N/A & N/A & 10.7 & $<0.0001$ & N/A & \\
\hline 1 year & $86.0 \pm 1.8$ & $90.8 \pm 0.9$ & $87.0 \pm 1.4$ & 4.8 & 0.0171 & -3.8 & 0.0224 & 1.0 & 0.6610 \\
\hline 2 years & $82.9 \pm 1.6$ & $89.2 \pm 1.1$ & $88.3 \pm 1.4$ & 6.3 & 0.0012 & -0.9 & 0.6132 & 5.4 & 0.0111 \\
\hline 3 years & $84.1 \pm 1.3$ & $89.4 \pm 1.5$ & $83.9 \pm 1.8$ & 5.3 & 0.0076 & -5.5 & 0.0189 & -0.2 & 0.9282 \\
\hline 4 years & $86.7 \pm 1.2$ & $86.5 \pm 1.8$ & $83.0 \pm 1.4$ & -0.2 & 0.9263 & -3.5 & 0.1248 & -3.7 & 0.0448 \\
\hline 5 years & $86.2 \pm 1.6$ & $90.9 \pm 1.3$ & $84.0 \pm 2.0$ & 4.7 & 0.0226 & -6.9 & 0.0038 & -2.2 & 0.3904 \\
\hline \multicolumn{10}{|c|}{ Flavored Milk } \\
\hline$<1$ year & N/A & $0.1 \pm 0.1$ & $1.2 \pm 0.5$ & N/A & N/A & 1.1 & 0.0310 & $\mathrm{~N} / \mathrm{A}$ & \\
\hline 1 year & $1.4 \pm 0.3$ & $1.8 \pm 0.6$ & $6.1 \pm 0.9$ & 0.4 & 0.5510 & 4.3 & 0.0001 & 4.7 & $<0.0001$ \\
\hline 2 years & $3.8 \pm 0.8$ & $2.2 \pm 0.6$ & $13.9 \pm 1.4$ & -1.6 & 0.1096 & 11.7 & $<0.0001$ & 10.1 & $<0.0001$ \\
\hline 3 years & $3.6 \pm 0.6$ & $4.6 \pm 1.1$ & $15.7 \pm 2.0$ & 1.0 & 0.4248 & 11.1 & $<0.0001$ & 12.1 & $<0.0001$ \\
\hline 4 years & $5.4 \pm 1.1$ & $3.2 \pm 0.8$ & $19.8 \pm 2.2$ & -2.2 & 0.1058 & 16.6 & $<0.0001$ & 14.4 & $<0.0001$ \\
\hline 5 years & $8.4 \pm 1.1$ & $12.3 \pm 1.9$ & $22.2 \pm 2.4$ & 3.9 & 0.0757 & 9.9 & 0.0012 & 13.8 & $<0.0001$ \\
\hline \multicolumn{10}{|c|}{ 100\% Fruit Juice } \\
\hline$<1$ year & $\mathrm{N} / \mathrm{A}$ & $31.5 \pm 2.4$ & $38.5 \pm 2.2$ & N/A & N/A & 7.0 & 0.0316 & $\mathrm{~N} / \mathrm{A}$ & \\
\hline 1 year & $36.0 \pm 2.3$ & $41.4 \pm 2.3$ & $63.3 \pm 2.2$ & 5.4 & 0.0969 & 21.9 & $<0.0001$ & 27.3 & $<0.0001$ \\
\hline 2 years & $36.1 \pm 1.9$ & $36.3 \pm 2.3$ & $61.3 \pm 2.4$ & 0.2 & 0.9465 & 25.0 & $<0.0001$ & 25.2 & $<0.0001$ \\
\hline 3 years & $32.0 \pm 2.2$ & $24.6 \pm 1.9$ & $54.7 \pm 2.7$ & -7.4 & 0.0109 & 30.1 & $<0.0001$ & 22.7 & $<0.0001$ \\
\hline 4 years & $35.1 \pm 2.1$ & $25.8 \pm 3.2$ & $50.9 \pm 2.7$ & -9.3 & 0.0151 & 25.1 & $<0.0001$ & 15.8 & $<0.0001$ \\
\hline 5 years & $32.1 \pm 2.4$ & $19.9 \pm 2.1$ & $41.7 \pm 3.6$ & -12.2 & 0.0001 & 21.8 & $<0.0001$ & 9.6 & 0.0265 \\
\hline \multicolumn{10}{|l|}{ Fruit Drink } \\
\hline$<1$ year & N/A & $2.9 \pm 0.6$ & $7.6 \pm 1.0$ & N/A & N/A & 4.7 & 0.0001 & N/A & \\
\hline 1 year & $33.4 \pm 2.7$ & $29.7 \pm 1.7$ & $27.2 \pm 1.4$ & -3.7 & 0.2462 & -2.5 & 0.2563 & -6.2 & 0.0415 \\
\hline 2 years & $40.5 \pm 2.5$ & $36.5 \pm 2.0$ & $35.5 \pm 2.2$ & -4.0 & 0.2115 & -1.0 & 0.7366 & -5.0 & 0.1348 \\
\hline 3 years & $41.2 \pm 2.2$ & $37.8 \pm 2.6$ & $38.7 \pm 2.4$ & -3.4 & 0.3181 & 0.9 & 0.7992 & -2.5 & 0.4426 \\
\hline 4 years & $40.9 \pm 2.1$ & $46.5 \pm 2.7$ & $42.3 \pm 3.0$ & 5.6 & 0.1016 & 4.2 & 0.2981 & 1.4 & 0.7022 \\
\hline 5 years & $41.8 \pm 2.7$ & $44.1 \pm 2.8$ & $51.7 \pm 2.7$ & 2.3 & 0.5543 & 7.6 & 0.0507 & 9.9 & 0.0095 \\
\hline \multicolumn{10}{|l|}{ Soft Drinks } \\
\hline$<1$ year & $\mathrm{N} / \mathrm{A}$ & $0.6 \pm 0.3$ & $2.4 \pm 0.6$ & N/A & N/A & 1.8 & 0.0073 & $\mathrm{~N} / \mathrm{A}$ & \\
\hline 1 year & $25.9 \pm 2.4$ & $22.7 \pm 1.5$ & $18.8 \pm 1.7$ & -3.2 & 0.2582 & -3.9 & 0.0854 & -7.1 & 0.0158 \\
\hline 2 years & $34.2 \pm 2.2$ & $37.1 \pm 2.1$ & $28.9 \pm 1.9$ & 2.9 & 0.3403 & -8.2 & 0.0038 & -5.3 & 0.0683 \\
\hline 3 years & $37.4 \pm 2.2$ & $40.3 \pm 3.2$ & $38.3 \pm 2.7$ & 2.9 & 0.4552 & -2.0 & 0.6329 & 0.9 & 0.7961 \\
\hline 4 years & $37.6 \pm 1.4$ & $43.0 \pm 2.7$ & $41.2 \pm 2.8$ & 5.4 & 0.0758 & -1.8 & 0.6435 & 3.6 & 0.2502 \\
\hline 5 years & $35.1 \pm 1.8$ & $46.3 \pm 2.2$ & $44.7 \pm 2.5$ & 11.2 & 0.0001 & -1.6 & 0.6309 & 9.6 & 0.0018 \\
\hline
\end{tabular}

*Data only available for children 6 months or older; N/A: not available.

${ }^{1}$ Mean \pm standard error; data represent percentage of children consuming any amount of described beverages in the 24-hr recall.

compared to NHANES 1976-1980 for nearly all ages. The trend was reversed for NHANES 1988-1994 versus NHANES 2001-2006, where fewer 1, 3 and 5 year olds were consuming milk. Age-related patterns for flavored milk consumption did not differ for the earlier two surveys but showed a significant increase $(\mathrm{p}<0.05)$ ranging from about $1 \%$ to $17 \%$, depending on age, during NHANES 2001-2006 compared to NHANES 1988-1994.
There was a small, but significant decline $(\mathrm{p}<0.05)$ in the percent of 3-5 year olds who consumed fruit juice during NHANES 1988-1994 compared to NHANES 1976-1980. In contrast, there was a significant increase $(\mathrm{p}<0.05)$ in the percent of all children from 1-5 years of age who consumed fruit juice in NHANES 2001-2006. There was a 22-30 percentage unit increase in fruit juice consumption in children 1 year and older. Fruit drink 
intake on the other hand, was not significantly different between the three decades with the exception of an increase in those less than 1 year of age. It was particularly evident in NHANES 1988-1994 and NHANES 20012006 that a greater percent of children consume soft drinks with increasing age. For example, in the most recent survey, approximately $19 \%$ consume soft drinks at 1 year of age, $29 \%$ at 2 years, $38 \%$ at 3 years, $41 \%$ at 4 years, and $45 \%$ at 5 years. Between NHANES 1976-1980 and NHANES 1988-1994, no clear age differences were present with the exception that there was an 11 percentage unit increase $(\mathrm{p}<0.001)$ in soft drinks consumption among 5 year olds in NHANES 1988-1994. Similarly, no significant age differences were found when NHANES 1988-1994 and NHANES 2001-2006 were compared, except for an increase $(\mathrm{p}<0.05)$ in children less than 1 year and a significant decrease in 2 year olds $(\mathrm{p}<0.05)$.

\section{Beverage amounts}

Overall for the group, there was a small decrease $(\mathrm{p}<0.05)$ in the amount of milk consumed between NHANES 19761980 and NHANES 1988-1994 and an increase $(\mathrm{p}<0.001)$ between NHANES 1988-1994 and NHANES 2001-2006 (Table 2). Among 1 year olds there was a small increase $(\mathrm{p}<0.05)$ in the mean amount consumed between NHANES 1976-1980 and NHANES 1988-1994, no significant change in 2 and 3 year olds, and a small to moderate decline in $4(\mathrm{p}<0.05)$ and $5(\mathrm{p}<0.001)$ year olds. In contrast there was a significant increase in the quantity of milk drank by $<1-5$ year olds when NHANES 2001-2006 was compared to NHANES 1988-1994. Milk consumption increased from 17.3 to $20.3 \mathrm{fl} \mathrm{oz} \mathrm{(}<<0.001$ ) in 1 year olds, 13.1 to $16.6 \mathrm{fl} \mathrm{oz}(\mathrm{p}<0.001)$ in 2 year olds, 13.3 to $14.9 \mathrm{fl} \mathrm{oz}$ $(\mathrm{p}<0.05)$ in 3 year olds, and 13.0 to $15.0 \mathrm{fl} \mathrm{oz}(\mathrm{p}<0.05)$ in 5 year olds. No significant changes were observed for 4 year olds. In the most recent survey, 2 and 3 year olds were consuming about 2 cups of milk a day and 4 and 5 year olds were consuming a little less than 2 cups a day.

Fruit juice consumption increased $(\mathrm{p}<0.001)$ in NHANES 1988-1994 compared to NHANES 19761980 and then remained stable through NHANES 20012006 for the entire sample. Children 1-3 years of age consumed significantly more fruit juice in NHANES 1988-1994 than in NHANES 1976-1980. Among 1, 2, and 3 year olds, mean fruit juice intake increased from 6.6 to $9.3 \mathrm{fl} \mathrm{oz}(\mathrm{p}<0.05), 8.3$ to $11.1 \mathrm{fl} \mathrm{oz}(\mathrm{p}<0.001)$, and 7.5 to $10.6 \mathrm{fl} \mathrm{oz}(\mathrm{p}<0.05)$, respectively. Changes were not significantly different for 4 and 5 year olds. The change in amounts consumed from NHANES 19881994 to 2001-2006 were not significantly different for any of the ages tested. During the NHANES 2001-2006 period, children less than 1 year were consuming $5.4 \mathrm{fl}$ oz, children 1-2 years were consuming about $11 \mathrm{fl} \mathrm{oz}$, children 3 years were consuming $12 \mathrm{fl} \mathrm{oz}$, and children
4-5 years were consuming about $10 \mathrm{fl} \mathrm{oz}$ on a daily basis.

The amount of fruit drink ingested did not change significantly for the entire group during the first two decades, but significantly increased $(\mathrm{p}<0.05)$ during the most recent one. None of the age groups demonstrated significant changes in the amount of fruit drink consumed between NHANES 1976-1980 and NHANES 1988-1994. Comparison of NHANES 1988-1994 to NHANES 2001-2006 indicated significant increases from 9.0 to $12.3 \mathrm{fl} \mathrm{oz}(\mathrm{p}<0.05)$ and 10.0 to $12.8 \mathrm{fl} \mathrm{oz}(\mathrm{p}<0.05)$ for 1 and 2 year olds, respectively. No changes between these surveys were observed for 3-5 year olds. The NHANES 2001-2006 survey found that children less than 1 year consumed $8.8 \mathrm{fl} \mathrm{oz}$ of fruit drink, and children 1-5 years consumed about 10$13 \mathrm{fl} \mathrm{oz/day.}$

Analysis of the group as a whole revealed a small increase $(\mathrm{p}<0.05)$ in the amount of soft drinks consumed between NHANES 1976-1980 and NHANES 1988-1994 as there were trends towards an increase among 3 and 5 years olds. No significant changes in the amounts of soft drinks consumed were noted between NHANES 19881994 and NHANES 2001-2006. During the most recent NHANES analysis, children less than 1 year, 1 year, 2 years, 3 years, 4 years, and 5 years consumed 1.8, 5.4, 6.2, 7.6, 8.8 and $9.8 \mathrm{fl} \mathrm{oz}$, respectively ( $\mathrm{p}$ for trend $<0.0001$ ). Increasing age was associated with increased amounts of soft drinks consumed.

\section{Beverage calorie and nutrient contributions}

Among the four main beverages consumed by children $<1-5$ years, milk was the largest daily beverage calorie contributor in all three decades surveyed, although there was a significant reduction in the last two surveys compared to NHANES 1976-1980 (Table 3). These calorie contributions include milk used on cereal or in food mixtures too. Milk was the primary contributor for all the macronutrients (Table 3) as well as calcium, phosphorus, magnesium, and potassium (Table 4). About $52 \%, 55 \%$, and $62 \%$ of the daily calcium intake were provided by milk in NHANES 2001-2006, NHANES 19881994, and NHANES 1976-1980, respectively. The reduced calcium intake from milk in the more recent surveys is consistent with reduced milk calories. Phosphorus intake from milk ranged from $37-42 \%$ across the three decades. Magnesium intake from milk was in the range of $27-28 \%$ in the last two surveys. Potassium intake from milk ranged from $31-37 \%$.

Percent daily caloric contribution of fruit juice and fruit drink was similar across all three surveys, in the range of $8-11 \%$. (Table 3) Fruit juice was an important provider (16-19\%) of potassium in the three surveys and magnesium (11\%) in the most recent survey (Table 4). Fruit drinks provided $5 \%$ or less of the daily intake of 
Table 2 Average amount of each beverage consumed by children <1-5 years during NHANES 1976-1980, NHANES 1988-1994, and NHANES 2001-2006

\begin{tabular}{|c|c|c|c|c|c|c|c|c|c|}
\hline \multirow[t]{2}{*}{ Beverage } & \multicolumn{3}{|c|}{ Mean Intake (FI. oz./day) ${ }^{1}$} & \multirow{2}{*}{$\begin{array}{l}\text { Change } \\
\text { (A-B) }\end{array}$} & \multirow{2}{*}{$\begin{array}{l}\text { p-value } \\
\text { (A-B) }\end{array}$} & \multirow{2}{*}{$\begin{array}{l}\text { Change } \\
\text { (B-C) }\end{array}$} & \multirow{2}{*}{$\begin{array}{l}\text { p-value } \\
\text { (B-C) }\end{array}$} & \multirow{2}{*}{$\begin{array}{l}\text { Change } \\
\text { (A-C) }\end{array}$} & \multirow{2}{*}{$\begin{array}{l}\text { p-value } \\
(\mathrm{A}-\mathrm{C})\end{array}$} \\
\hline & $\begin{array}{l}\text { NHANES } \\
1976-1980^{*}(\mathrm{~A})\end{array}$ & $\begin{array}{l}\text { NHANES } \\
\text { 1988-1994 (B) }\end{array}$ & $\begin{array}{l}\text { NHANES } \\
2001-2006(C) \\
\end{array}$ & & & & & & \\
\hline \multicolumn{10}{|l|}{ Total Milk } \\
\hline$<1-5$ years & $14.7 \pm 0.2$ & $14.0 \pm 0.2$ & $16.3 \pm 0.4$ & -0.7 & 0.0133 & 2.3 & $<0.0001$ & 1.6 & 0.0003 \\
\hline$<1$ year & N/A & $22.0 \pm 3.4$ & $17.8 \pm 1.2$ & N/A & N/A & -4.2 & 0.2441 & N/A & \\
\hline 1 year & $15.3 \pm 0.5$ & $17.3 \pm 0.6$ & $20.3 \pm 0.5$ & 2.0 & 0.0104 & 3 & 0.0001 & 5 & $<0.0001$ \\
\hline 2 years & $12.6 \pm 0.4$ & $13.1 \pm 0.4$ & $16.6 \pm 0.5$ & 0.5 & 0.3768 & 3.5 & $<0.0001$ & 4 & $<0.0001$ \\
\hline 3 years & $13.5 \pm 0.4$ & $13.3 \pm 0.5$ & $14.9 \pm 0.6$ & -0.2 & 0.7548 & 1.6 & 0.0405 & 1.4 & 0.0522 \\
\hline 4 years & $14.5 \pm 0.4$ & $13.2 \pm 0.4$ & $13.9 \pm 0.4$ & -1.3 & 0.0216 & 0.7 & 0.2159 & -0.6 & 0.2888 \\
\hline 5 years & $15.3 \pm 0.5$ & $13.0 \pm 0.4$ & $15.0 \pm 0.7$ & -2.3 & 0.0003 & 2 & 0.0131 & -0.3 & 0.7273 \\
\hline \multicolumn{10}{|c|}{ 100\% Fruit Juice } \\
\hline$<1-5$ years & $7.4 \pm 0.2$ & $9.5 \pm 0.4$ & $10.3 \pm 0.3$ & 2.1 & $<0.0001$ & 0.8 & 0.1096 & 2.9 & $<0.0001$ \\
\hline$<1$ year & N/A & $4.8 \pm 0.3$ & $5.4 \pm 0.3$ & N/A & N/A & 0.6 & 0.1573 & N/A & \\
\hline 1 year & $6.6 \pm 0.3$ & $9.3 \pm 0.8$ & $10.8 \pm 0.5$ & 2.7 & 0.0016 & 1.5 & 0.1118 & 4.2 & $<0.0001$ \\
\hline 2 years & $8.3 \pm 0.4$ & $11.1 \pm 0.6$ & $11.0 \pm 0.5$ & 2.8 & 0.0001 & -0.1 & 0.8981 & 2.7 & $<0.0001$ \\
\hline 3 years & $7.5 \pm 0.4$ & $10.6 \pm 1.2$ & $12.2 \pm 0.8$ & 3.1 & 0.0143 & 1.6 & 0.2673 & 4.7 & $<0.0001$ \\
\hline 4 years & $8.0 \pm 0.5$ & $8.8 \pm 0.5$ & $9.7 \pm 0.4$ & 0.8 & 0.2579 & 0.9 & 0.1599 & 1.7 & 0.0079 \\
\hline 5 years & $8.1 \pm 0.5$ & $8.2 \pm 0.7$ & $10.0 \pm 0.6$ & 0.1 & 0.9075 & 1.8 & 0.0509 & 1.9 & 0.0150 \\
\hline \multicolumn{10}{|l|}{ Fruit Drink } \\
\hline$<1-5$ years & $10.4 \pm 0.3$ & $10.7 \pm 0.3$ & $12.1 \pm 0.4$ & 0.3 & 0.4795 & 1.4 & 0.0051 & 1.7 & 0.0007 \\
\hline$<1$ year & N/A & $8.1 \pm 2.5$ & $8.8 \pm 1.2$ & N/A & N/A & 0.7 & 0.8007 & N/A & \\
\hline 1 year & $8.8 \pm 0.6$ & $9.0 \pm 0.6$ & $12.3 \pm 0.9$ & 0.2 & 0.8137 & 3.3 & 0.0023 & 3.5 & 0.0012 \\
\hline 2 years & $10.8 \pm 0.5$ & $10.0 \pm 0.7$ & $12.8 \pm 0.8$ & -0.8 & 0.3524 & 2.8 & 0.0084 & 2 & 0.0340 \\
\hline 3 years & $10.5 \pm 0.6$ & $10.7 \pm 0.7$ & $10.1 \pm 0.4$ & 0.2 & 0.8283 & -0.6 & 0.4568 & -0.4 & 0.5791 \\
\hline 4 years & $11.1 \pm 0.6$ & $10.6 \pm 0.6$ & $12.2 \pm 0.9$ & -0.5 & 0.5557 & 1.6 & 0.1391 & 1.1 & 0.3092 \\
\hline 5 years & $11.1 \pm 0.5$ & $12.7 \pm 0.8$ & $13.1 \pm 0.9$ & 1.6 & 0.0899 & 0.4 & 0.7398 & 2 & 0.0521 \\
\hline \multicolumn{10}{|l|}{ Soft Drinks } \\
\hline$<1-5$ years & $7.0 \pm 0.3$ & $7.8 \pm 0.2$ & $7.9 \pm 0.4$ & 0.8 & 0.0265 & 0.1 & 0.8231 & 0.9 & 0.0719 \\
\hline$<1$ year & N/A & $6.4 \pm 2.8$ & $1.8 \pm 0.4$ & N/A & N/A & -4.6 & 0.1013 & $\mathrm{~N} / \mathrm{A}$ & \\
\hline 1 year & $5.4 \pm 0.4$ & $5.0 \pm 0.3$ & $5.4 \pm 1.4$ & 0.4 & 0.4237 & 0.4 & 0.7800 & 0 & 1.0000 \\
\hline 2 years & $6.6 \pm 0.3$ & $6.7 \pm 0.3$ & $6.2 \pm 0.3$ & 0.1 & 0.8137 & -0.5 & 0.2386 & -0.4 & 0.3458 \\
\hline 3 years & $6.8 \pm 0.3$ & $7.7 \pm 0.4$ & $7.6 \pm 0.6$ & 0.9 & 0.0719 & 0.1 & 0.8897 & 0.8 & 0.2330 \\
\hline 4 years & $7.9 \pm 0.4$ & $8.4 \pm 0.4$ & $8.8 \pm 0.5$ & 0.5 & 0.3768 & 0.4 & 0.5322 & 0.9 & 0.1599 \\
\hline 5 years & $8.4 \pm 0.6$ & $9.7 \pm 0.5$ & $9.8 \pm 0.6$ & 1.3 & 0.0960 & 0.1 & 0.8981 & 1.4 & 0.0990 \\
\hline
\end{tabular}

${ }^{1}$ Mean \pm standard error; fluid ounce (fl. oz.). calculated on the assumption that $1 \mathrm{fl} \mathrm{oz}=30 \mathrm{~g}$; N/A: not available; $8 \mathrm{fl}$. oz. is equivalent to one cup.

*Data only available for children 6 months or older.

calcium, phosphorus, magnesium, or potassium in all three surveys (Table 4). The caloric contribution of soft drinks in the range of $5-6 \%$ did not significantly differ among the three surveys (Table 3). Soft drinks provided very little of the nutrients evaluated (Table 4). The combined calorie contribution from fruit juice, fruit drinks and soft drinks over this thirty year period has increased, but given the parallel rise in total calorie intake the proportion from these beverages has remained relatively consistent at about a quarter of total daily calorie intake
(1976-1980: 324 kcals, 23\% of total kcal intake; 19881994: 358 kcals, 25\% of total kcal intake; 2001-2006: 384 kcals, $25 \%$ of total kcal intake; data not shown).

Use of different types of milks (Figures 2 and 3) and eating occasion (data not shown) for milk were evaluated in NHANES 1988-94 and NHANES 2001-2006. In both surveys, whole milk was the predominant milk introduced at less than 1 year of age and consumed at 1 year of age. Whole milk intake declined with increasing age in NHANES 2001-2006 compared to NHANES 1988-1994. 
Table 3 Energy and macronutrient intake contributed by milk, fruit juice, fruit drink, and soft drinks in the diets of children <1-5 years participating in NHANES 1976-1980, NHANES 1988-1994, and NHANES 2001-2006

\begin{tabular}{|c|c|c|c|c|c|c|c|c|}
\hline \multirow[t]{2}{*}{ Nutrient/Survey } & \multicolumn{2}{|l|}{ Milk } & \multicolumn{2}{|c|}{$100 \%$ Fruit Juice } & \multicolumn{2}{|l|}{ Fruit Drink } & \multicolumn{2}{|l|}{ Soft Drinks } \\
\hline & Mean \pm S.E. & \% Daily Intake & Mean \pm S.E. & $\%$ Daily Intake & Mean \pm S.E. & \% Daily Intake & Mean \pm S.E. & \% Daily Intake \\
\hline \multicolumn{9}{|l|}{ Calories (kcal/d) } \\
\hline NHANES 1976-1980 & $281 \pm 5$ & $20.8^{a}$ & $114 \pm 3$ & $8.4^{a}$ & $128 \pm 4$ & $9.0^{a}$ & $82 \pm 3$ & $5.9^{a}$ \\
\hline NHANES 1988-1994 & $234 \pm 4$ & $16.8^{b}$ & $143 \pm 6$ & $10.6^{b}$ & $129 \pm 4$ & $8.7^{a}$ & $86 \pm 3$ & $5.9^{a}$ \\
\hline NHANES 2001-2006 & $279 \pm 6$ & $18.7^{c}$ & $149 \pm 5$ & $9.8^{c}$ & $150 \pm 5$ & $9.4^{b}$ & $85 \pm 4$ & $5.3^{a}$ \\
\hline \multicolumn{9}{|l|}{ Total Fat (g/d) } \\
\hline NHANES 1976-1980 & $13.5 \pm 0.2$ & $25.8^{a}$ & $0.2 \pm 0.0$ & $0.6^{a}$ & $0.0 \pm 0.0$ & 0.0 & $0.0 \pm 0.0$ & 0.0 \\
\hline NHANES 1988-1994 & $10.9 \pm 0.2$ & $21.4^{b}$ & $0.3 \pm 0.0$ & $0.8^{b}$ & $0.0 \pm 0.0$ & 0.1 & $0.0 \pm 0.0$ & 0.0 \\
\hline NHANES 2001-2006 & $12.0 \pm 0.3$ & $22.5^{b}$ & $0.4 \pm 0.0$ & $0.7^{\mathrm{ab}}$ & $0.1 \pm 0.0$ & 0.2 & $0.0 \pm 0.0$ & 0.0 \\
\hline \multicolumn{9}{|l|}{ Saturated Fat $(\mathrm{g} / \mathrm{d})$} \\
\hline NHANES 1976-1980 & $7.5 \pm 0.1$ & $26.3^{a}$ & $0.0 \pm 0.0$ & 0 & $0.0 \pm 0.0$ & 0.0 & $0.0 \pm 0.0$ & 0.0 \\
\hline NHANES 1988-1994 & $6.8 \pm 0.2$ & $32.0^{b}$ & $0.0 \pm 0.0$ & 0.3 & $0.0 \pm 0.0$ & 0.1 & $0.0 \pm 0.0$ & 0.0 \\
\hline NHANES 2001-2006 & $7.2 \pm 0.2$ & $32.8^{b}$ & $0.1 \pm 0.0$ & 0.4 & $0.0 \pm 0.0$ & 0.0 & $0.0 \pm 0.0$ & 0.0 \\
\hline \multicolumn{9}{|l|}{ Protein $(\mathrm{g} / \mathrm{d})$} \\
\hline NHANES 1976-1980 & $16.3 \pm 0.3$ & $31.4^{a}$ & $1.3 \pm 0.1$ & $2.9^{a}$ & $0.0 \pm 0.0$ & 0.0 & $0.0 \pm 0.0$ & 0.0 \\
\hline NHANES 1988-1994 & $13.8 \pm 0.2$ & $26.5^{b}$ & $0.5 \pm 0.0$ & $1.2^{b}$ & $0.1 \pm 0.0$ & 0.2 & $0.0 \pm 0.0$ & 0.0 \\
\hline NHANES 2001-2006 & $15.9 \pm 0.4$ & $29.1^{c}$ & \pm 0.0 & $2.0^{c}$ & $0.1 \pm 0.0$ & 0.3 & $0.1 \pm 0.0$ & 0.2 \\
\hline
\end{tabular}

For each nutrient, different subscripts down a column $(a, b, c)$ indicate statistically significant differences $(p<0.05)$.

Two percent or reduced-fat milk was consumed in almost equivalent amounts by age 5 in the most recent survey. One-percent milk and skim milk were consumed in very low amounts for all the years surveyed. Breakfast was the eating occasion where milk was most consumed in both NHANES 1988-1994 and NHANES
2001-2006. Snack time was the next meal occasion in which milk was consumed in both surveys. Slightly less milk was consumed at lunch or dinner than at other meal times. In the context of total dairy intake, relatively small amounts of milk are consumed during meal occasions.

Table 4 Calcium, phosphorus, magnesium, and potassium intake contributed by milk, fruit juice, fruit drink, and soft drinks in the diets of children <1-5 years participating in NHANES 1976-1980, NHANES 1988-1994, and NHANES 2001-2006

\begin{tabular}{|c|c|c|c|c|c|c|c|c|}
\hline \multirow[t]{2}{*}{ Nutrient/Survey } & \multicolumn{2}{|l|}{ Milk } & \multicolumn{2}{|c|}{$100 \%$ Fruit Juice } & \multicolumn{2}{|l|}{ Fruit Drink } & \multicolumn{2}{|l|}{ Soft Drinks } \\
\hline & Mean \pm S.E. & \% Daily Intake & Mean \pm S.E. & $\%$ Daily Intake & Mean \pm S.E. & $\%$ Daily Intake & Mean \pm S.E. & \% Daily Intake \\
\hline \multicolumn{9}{|l|}{ Calcium (mg/d) } \\
\hline NHANES 1976-1980 & $553 \pm 10$ & $61.7^{a}$ & $20.6 \pm 0.7$ & $3.7^{a}$ & $29.1 \pm 2.2$ & $4.7^{a}$ & $0.0 \pm 0.0$ & $0.0^{a}$ \\
\hline NHANES 1988-1994 & $504 \pm 8$ & $54.5^{b}$ & $21.8 \pm 1.0$ & $3.5^{a}$ & $31.5 \pm 1.5$ & $4.9^{a}$ & $6.9 \pm 0.0$ & $1.3^{b}$ \\
\hline NHANES 2001-2006 & $565 \pm 13$ & $52.3^{c}$ & $55.2 \pm 4.0$ & $5.7^{b}$ & $34.7 \pm 1.9$ & $4.9^{\mathrm{a}}$ & $5.8 \pm 0.3$ & $1.0^{b}$ \\
\hline \multicolumn{9}{|l|}{ Phosphorus (mg/d) } \\
\hline NHANES 1976-1980 & $435 \pm 8$ & $41.8^{a}$ & $32.8 \pm 1.2$ & $4.0^{a}$ & $42.9 \pm 3.0$ & $4.7^{a}$ & $11.0 \pm 1.4$ & $1.5^{\mathrm{a}}$ \\
\hline NHANES 1988-1994 & $396 \pm 7$ & $37.1^{\mathrm{b}}$ & $22.0 \pm 0.9$ & $2.8^{b}$ & $26.6 \pm 1.8$ & $3.0^{b}$ & $17.1 \pm 0.9$ & $2.0^{b}$ \\
\hline NHANES 2001-2006 & $456 \pm 10$ & $39.7^{c}$ & $31.6 \pm 1.1$ & $3.2^{b c}$ & $11.5 \pm 0.7$ & $1.5^{c}$ & $11.8 \pm 0.9$ & $1.8^{b}$ \\
\hline \multicolumn{9}{|l|}{ Magnesium (mg/d) } \\
\hline NHANES 1976-1980 & $\mathrm{N} / \mathrm{A}$ & N/A & N/A & $\mathrm{N} / \mathrm{A}$ & $\mathrm{N} / \mathrm{A}$ & $\mathrm{N} / \mathrm{A}$ & $\mathrm{N} / \mathrm{A}$ & $\mathrm{N} / \mathrm{A}$ \\
\hline NHANES 1988-1994 & $56.2 \pm 0.9$ & $27.8^{a}$ & $12.9 \pm 0.6$ & $7.1^{a}$ & $5.2 \pm 0.2$ & $3.1^{\mathrm{a}}$ & $2.3 \pm 0.1$ & $1.5^{\mathrm{a}}$ \\
\hline NHANES 2001-2006 & $53.7 \pm 1.3$ & $27.4^{a}$ & $22.0 \pm 0.8$ & $11.1^{b}$ & $7.6 \pm 0.3$ & $4.9^{b}$ & $1.7 \pm 0.1$ & $1.1^{b}$ \\
\hline \multicolumn{9}{|l|}{ Potassium (mg/d) } \\
\hline NHANES 1976-1980 & $679 \pm 13$ & $36.9^{a}$ & $386 \pm 14$ & $18.8^{a}$ & $26.2 \pm 3.4$ & $1.7^{\mathrm{a}}$ & $0.0 \pm 0.0$ & $0.0^{a}$ \\
\hline NHANES 1988-1994 & $645 \pm 11$ & $31.4^{b}$ & $332 \pm 14$ & $16.1^{\mathrm{b}}$ & $43.8 \pm 2.2$ & $2.7^{b}$ & $2.6 \pm 0.3$ & $0.2^{a}$ \\
\hline NHANES 2001-2006 & $727 \pm 17$ & $33.5^{c}$ & $436 \pm 14$ & $19.2^{a}$ & $74.9 \pm 3.0$ & $4.5^{c}$ & $4.0 \pm 0.3$ & $0.3^{a}$ \\
\hline
\end{tabular}

For each nutrient, different subscripts $(a, b)$ for each survey indicate statistically significant differences $(p<0.05)$. 


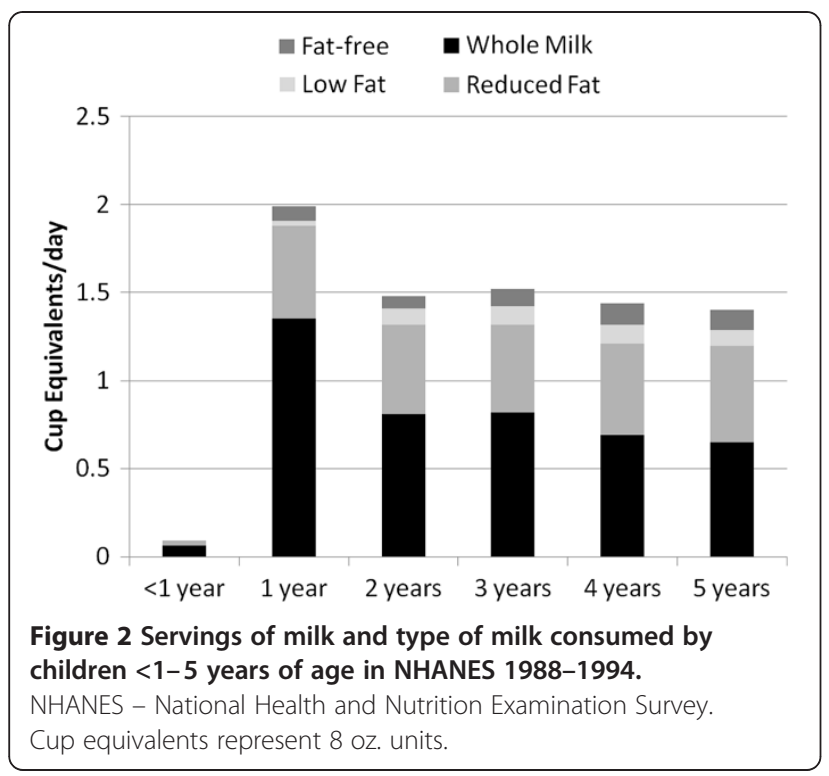

\section{Discussion}

The 2005 Dietary Guidelines for Americans indicated that the nutrients of concern for children were vitamin E, calcium, magnesium, potassium, and dietary fiber [22]. Beverages are a potential source of calcium, magnesium, and potassium, hence these nutrients were evaluated in our study, along with phosphorus, a non-fortified nutrient provided by milk. After our analysis was completed, the 2010 Dietary Guidelines for Americans were released, which noted vitamin $\mathrm{D}$, calcium, potassium, and dietary fiber were nutrients of concern for children above the age of 2 [6]. Milk is the primary source of vitamin D in children's diets, but it was not possible to

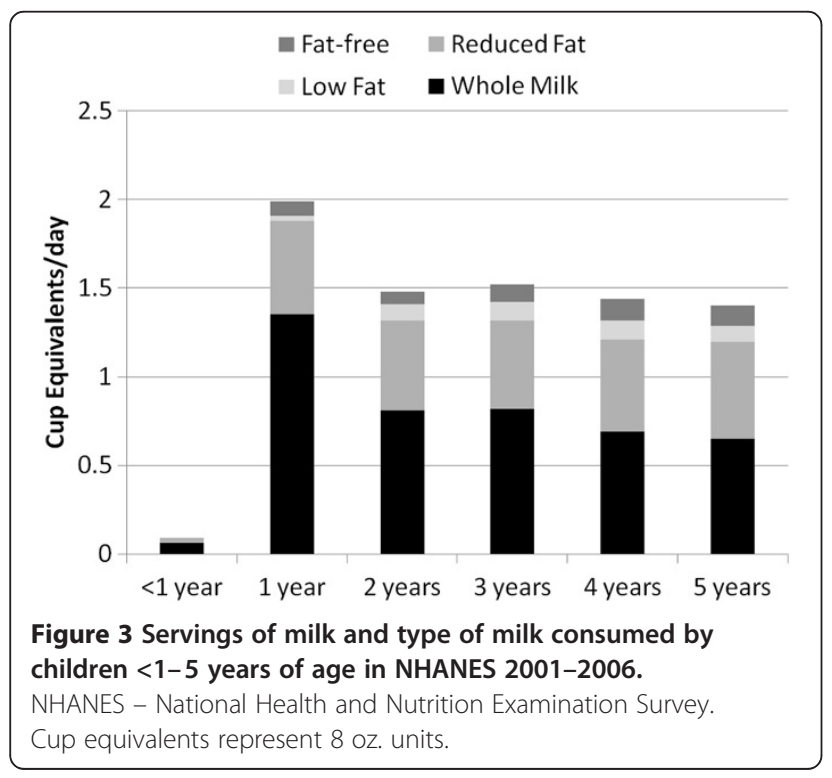

evaluate vitamin $\mathrm{D}$ trends because vitamin $\mathrm{D}$ intake was not assessed in NHANES prior to 2002.

Milk has been and still is the beverage consumed by most preschool children. However, there was a trend towards fewer children consuming milk in the last decade as evidence by this data and previous research [13]. Only $77 \%$ of children $<1-5$ years consumed milk of any type during NHANES 2001-2006 compared to $84-85 \%$ in NHANES 1988-1994 and NHANES 1976-1980. To ensure optimal growth and health, the 2010 Dietary Guidelines for Americans recommends children aged 2-3 years drink 2 cups (16 oz) and children aged 4-8 years drink $2 \frac{1}{2}$ cups $(20 \mathrm{fl} \mathrm{oz})$ of fat-free or low-fat milk or equivalent dairy servings a day [6]. On average 2-3 year olds in NHANES 2001-2006 consumed the recommended amount of milk daily. However, children 4 and 5 years of age were consuming $14-15 \mathrm{fl} \mathrm{oz} /$ day; to meet the Dietary Guidelines, recommendation through fluid milk intake alone, $20 \mathrm{fl} \mathrm{oz} /$ day would need to be consumed for this age group. Data from Krebs-Smith, et al. indicate about one out of four young children 2-8 years old do not meet milk group recommendations [23]. Although the Dietary Guidelines recommend everyone 2 years of age or older consume low-fat (i.e., 1\%) or fatfree milk; the majority of milk currently consumed by $2-5$ year olds was whole or $2 \%$ milk. Several studies have shown potential health benefits of starting consumption of low-fat/fat-free milk early in life $[24,25]$ and reducedfat milk would be appropriate for overweight or obese children between 12 months and 2 years of age or those with a family history of obesity, dyslipidemia, or cardiovascular disease [26]. Milk consumption has been reported to increase the likelihood of achieving the recommended intake of calcium by $37 \%$ and increase intakes of vitamin $\mathrm{A}$, folate, vitamin B12, and magnesium in 2-5 year olds participating in CSFII 1994-96 [14]. This study found that in addition to calcium and magnesium, milk is a major contributor to the daily intake of phosphorus and potassium in young children. Research has shown young children who drink flavored milk have comparable or higher intakes of many of these nutrients compared to those who exclusively drink plain milk, in part because flavored milk consumers have higher total milk intakes [27].

Over $50 \%$ of children $1-5$ years consumed fruit juice in NHANES 2001-2006, representing an increase of $23 \%$ from the previous decade. Wang et al. [13] found a non-significant increase from $41 \%$ to $46 \%$ from NHANES 1988-1994 to NHANES 1999-2004 in their study of children 2-5 years of age. Fruit juice was an important source of potassium and magnesium in the diets of these children and has been positively linked to achieving recommended intakes of vitamin $\mathrm{C}$ and folate in 2-5 year old children in previous research [14]. Mean 
daily fruit juice consumption in 2001-2006 was $5.4 \mathrm{fl}$ oz/day for children less than 1 year and 10-12 fl oz/day for children 1-5 years. For the entire group, mean intake was $10.3 \mathrm{fl} \mathrm{oz/day}$ in NHANES 2001-2006 compared to 9.5 and $7.4 \mathrm{fl} \mathrm{oz/day}$ in NHANES 1988-1994 and NHANES 1976-1980, respectively. Consistent with our findings, Wang et al. [13] observed a significant increase from $9.9 \mathrm{fl} \mathrm{oz} /$ day in NHANES 1988-1994 to $11.1 \mathrm{fl} \mathrm{oz/}$ day in NHANES 1999-2004 in their preschool sample. This is higher than the fruit juice amount recommended by the AAP, American Heart Association, and Dietary Guidelines (4-6 fl oz/day), and some children were introduced to fruit juice or drinks before the recommended 6 months of age $[5,6,28]$.

In the last three decades, at least $35 \%$ of young children consumed fruit drinks, which is similar to the percentage reported by other studies $[14,20]$. The data indicate a significant increase in the amount of fruit drinks consumed in the last decade, particularly in children 1 and 2 years of age. Children less than 1 year consumed $8.8 \mathrm{fl} \mathrm{oz} /$ day and children $1-5$ years consumed 10-13 fl oz/day in this analysis of NHANES 2001-2006, which was higher than estimates in CSFII 1994-1996, 1998 (4 fl oz/day) for children 6 months to 6 years [28] and $8 \mathrm{fl} \mathrm{oz/day} \mathrm{in} \mathrm{CSFII} \mathrm{1994-1996} \mathrm{for} \mathrm{children} \mathrm{2-5}$ years. The contribution of calcium, phosphorus, magnesium, and potassium from fruit drinks was negligible. Generally, the amount of fruit drinks consumed was greater than fruit juice for children of all ages analyzed, yet the caloric contribution of fruit drink and fruit juice to these children's diets was similar. Across all three decades, fruit drinks provided considerably more calories in the diets of preschool children than soft drinks. Although fruit drinks were not a source of the nutrients we measured, many fruit drinks are fortified with vitamin $\mathrm{C}$ which was positively associated with achieving recommended intakes of vitamin $\mathrm{C}$ in the study by Ballew et al. [14]. Most fruit drinks contain 10\% or less fruit juice and a substantial amount of added sugars.

Approximately one-third of young children consumed soft drinks in all three NHANES analyses. These results are consistent with some previous from CSFII 19941996 [14], but lower than others including studies of NHANES 1999-2002 (39\% 2-5 year olds) [20] and CSFII 1994-1995 (51\%) [29]. As children age from 1 year to 5 years, a greater percentage of the population consumed soft drinks. In NHANES 2001-2006, 19\% of 1 year olds consumed soft drinks, which increased to $45 \%$ by age 5 years. The mean amount of soft drinks marginally increased from $7 \mathrm{fl} \mathrm{oz/day}$ in NHANES 1976-1980 to $7.8 \mathrm{fl} \mathrm{oz/day} \mathrm{in} \mathrm{NHANES} \mathrm{1988-1994} \mathrm{and}$ $7.9 \mathrm{fl} \mathrm{oz/day}$ in NHANES 2001-2006. These findings are similar to data from CSFII 1994-1996 [14] but appreciably higher than $2.9 \mathrm{fl} \mathrm{oz} /$ day indicated by Rampersaud et al. [29] for CSFII 1994-1996, 1998. Carbonated beverages were negatively associated with meeting the recommended levels of vitamin $\mathrm{A}$, vitamin $\mathrm{C}$, and calcium [14].

The cumulative consumption of beverages with added sugar has been inversely linked to overall diet quality and meeting the adequacy of several nutrients [15]. In our analysis of the most recent NHANES, an additional 85 empty calories or $5 \%$ of total daily intake was consumed as soft drinks. The decline in milk consumption and the steady intake of nutrient poor beverages such as fruit drinks and soft drinks in our analysis is corroborated by others. Carbonated beverages and added sugar juice drinks were inversely associated with milk intakes in children 1-5 years of age in the Iowa Fluoride Study [15]. Harnack et al. [30] reported in their analysis of CSFII 1994 that high soft drink consumption appears to displace milk and fruit juice in the diets of preschool aged children. For example, those that consumed $9 \mathrm{fl} \mathrm{oz}$ or more soft drinks per day were 3.8 times more likely to consume less than $8 \mathrm{oz}$ of milk per day compared to those that did not drink any soft drinks. Although the issue of displacement of milk by other beverages cannot be specifically addressed in cross-sectional data, evidence from longitudinal studies validates this phenomenon. Fiorito and colleagues [31] followed nonHispanic white girls from age 5-15 years starting in 1996 and observed that early differences in carbonated beverage intake were predictive of later carbonated beverage and milk intake as well as selected nutrients. Girls who consumed carbonated beverages at age 5 years had higher intakes of carbonated beverages, lower milk intake, higher intake of added sugars, lower protein, fiber, vitamin D, calcium, magnesium, phosphorous, and potassium for the 10 years of follow-up. Another study of non-Hispanic white girls who were followed from age 5-9 years observed that girls who consumed adequate amounts of calcium consumed on average twice as much milk, had smaller decreases in milk intake, and consumed 18\% less sweetened beverages [32].

The link between beverage consumption, energy intake and body weight have been evaluated in this young population, and is a significant concern because obesity tends to track over time [33]. High intake of sweetened beverages is linked to increased energy intake, weight gain and adiposity in children. Regular consumption of sweetened beverages between meals by children $2.5-4.5$ years more than doubled the odds of being overweight at 4.5 years [12]. In a longitudinal study of girls, sweetened beverage consumption (sodas, sport drinks, fruit drinks, and sweetened coffee or tea) at age 5, but not milk or $100 \%$ juice, predicted adiposity in childhood and adolescence [19]. Higher intake of sweetened beverages at 5 years was correlated with a higher weight, percent 
of body fat, and waist circumference from 5-15 years of age [19]. Sweetened beverages in both of these longitudinal studies did not include flavored milk but included sugar-sweetened or artificially sweetened fruit drinks and carbonated beverages. Excessive fruit juice consumption in preschool children has been associated with obesity in some [34] but not all studies [11,35]. Wiley [36] found milk intake was positively associated with BMI among children aged 2-4 years in an analysis of NHANES 19992004 data while a cross-sectional analysis of NHANES 1999-2002 data of 2-5 year olds revealed that energy intakes of flavored milk drinkers were higher than those that consumed plain milk or non milk drinkers, but BMI indices did not differ among the three groups [27]. Huh et al. [37] reported in their study that milk intake at age 2 years, whether full or reduced-fat, was not linked to the risk of overweight at age 3 years.

Our analysis was limited by the use of cross-sectional data and associated analytical data across three time periods. But in the case of nationally, representative dietary data, NHANES data is the only source currently available. Given the cross-sectional approach of NHANES causality cannot be determined. Also the data was based on a single 24-hour dietary recall which may not be a fair representation of an individual's usual intake. The 24-hour dietary recall has been shown to be prone to both under- an over-reporting. Nevertheless, this method has been shown to be valid in estimating the mean intake of a population [38,39].

In summary, the number of young children consuming milk has significantly declined while those consuming fruit juice has increased dramatically in the last decade compared to the previous two decades. The proportion of children that consume fruit drinks and soft drinks has remained high and relatively stable across the three decade time period. Milk is the main source of calcium in the diets of these children, which has been declining alongside increased reduced-fat milk consumption. Milk is also a major source of magnesium and potassium, short-fall nutrients identified by the 2005 and 2010 Dietary Guidelines for Americans. The amount of milk that is consumed in this age group is less than recommended, particularly among 4 and 5 year olds, where fruit juice consumption is significantly higher than recommended. Fruit drinks are a significant source of calories. A greater number of children consume soft drinks as they age from 1-5 years. Since dietary patterns established as young children are carried throughout childhood and adolescence, and a link between non-milk sweetened beverages and obesity has been increasingly demonstrated, it is prudent that parents, educators and child caretakers replace some of the nutrient poor beverages young children are currently consuming with low-fat and fat-free milk.

\section{Competing interests}

VLF as Senior Vice President of Nutrition Impact, LLC, performs consulting and database analyses for various food and beverage companies and related entities. EEQ is an employee of Dairy Research Institute/National Dairy Council.

\section{Authors' contributions}

VLF designed the study, was primarily responsible for the data analysis, and provided critical input into the manuscript; EEQ helped with data analysis and drafted the manuscript. All authors read and approved the final manuscript.

\section{Sources of support}

Dairy Research Institute administered by the National Dairy Council

\section{Author details}

${ }^{1}$ Nutrition Impact, LLC, 9725 D Drive North, Battle Creek, Ml 49104, USA. ${ }^{2}$ Dairy Research Institute/National Dairy Council, Rosemont, IL 60018, USA.

Received: 31 December 2011 Accepted: 24 October 2012

Published: 31 October 2012

\section{References}

1. Birch L, Savage JS, Ventura A: Influences on the development of children's eating behaviors: from infancy to adolescence. Can J Diet Pract Res 2007, 68:s1-s56.

2. Savage JS, Fisher JO, Birch LL: Parental influence on eating behavior: conception to adolescence. J Law Med Ethics 2007, 35:22-34.

3. Gartner LM, Morton J, Lawrence RA, Naylor AJ, O'Hare D, Schanler RJ, Eidelman Al: American Academy of Pediatrics Section on Breastfeeding. Breastfeeding and the use of human milk. Pediatrics 2005, 115:496-506.

4. Committee on Nutrition and the Council on Sports Medicine and Fitness: Sports drinks and energy drinks for children and adolescents: are they appropriate? Pediatrics 2011, 127:1182-1189.

5. American Academy of Pediatrics Committee on Nutrition: The use and misuse of fruit juice in pediatrics. Pediatrics 2001, 107:1210-1213.

6. U.S. Department of Health and Human Services/ U.S. Department of Agriculture: Dietary Guidelines for Americans; 2010. http://www.cnpp.usda. gov/DGAs2010-PolicyDocument.htm (accessed February 2011).

7. Skinner JD, Ziegler P, Ponza M: Transitions in infants' and toddlers' beverage patterns. J Am Diet Assoc 2004, 104(1 Suppl):s45-s50.

8. Popkin BM: Patterns of beverage use across the lifecycle. Physiol Behav 2010, 100:4-9

9. Wiley AS: Consumption of milk, but not other dairy products, is associated with height among US preschool children in NHANES 19992002. Ann Hum Biol 2009, 36:125-138.

10. Lee WT, Leung SS, Lui SS, Lau J: Relationship between long-term calcium intake and bone mineral content of children aged from birth to 5 years. Br J Nutr 1993, 70:235-248.

11. Skinner JD, Carruth BR: A longitudinal study of children's juice intake and growth: the juice controversy revisited. J Am Diet Assoc 2001, 101:432-437.

12. Dubois L, Farmer A, Girard M, Peterson K: Regular sugar-sweetened beverage consumption between meals increases risk of overweight among preschool-aged children. J Am Diet Assoc 2007, 107:924-935.

13. Wang YC, Bleich SN, Gortmaker SL: Increasing caloric contribution from sugar-sweetened beverages and $100 \%$ fruit juices among US children and adolescents, 1988-2004. Pediatrics 2008, 121(6):e1604-e1614.

14. Ballew C, Kuester S, Gillespie C: Beverage choices affect adequacy of children's nutrient intakes. Arch Pediatr Adolesc Med 2000, 154:1148-1152.

15. Marshall, Marshall TA, Eichenberger Gilmore JM, Broffitt B, Stumbo PJ, Levy SM: Diet quality in young children is influenced by beverage consumption. J Am Coll Nutr 2005, 24:65-75.

16. Keller KL, Kirzner J, Pietrobelli A, St-Onge MP, Faith MS: Increased sweetened beverage intake is associated with reduced milk and calcium intake in 3- to 7-year-old children at multi-item laboratory lunches. J Am Diet Assoc 2009, 109:497-501.

17. Ogden C, Carrroll M: CDC (Centers for Disease Control): NCHS Health E-Stat. Prevalence of Obesity Among Children and Adolescents: United States, Trends 1963-1965 Through 2007-2008. [http://www.cdc.gov/nchs/data/ hestat/obesity_child_07_08/obesity_child_07_08.htm\#table 1] (accessed December 2010). 
18. Vartanian $L R$, Schwartz MB, Brownell KD: Effects of soft drink consumption on nutrition and health: a systematic review and meta-analysis. Am J Public Health 2007, 97:667-675.

19. Fiorito LM, Marini M, Francis LA, Smiciklas-Wright $H$, Birch L: Beverage intake of girls at age 5 y predicts adiposity and weight status in childhood and adolescence. Am J Clin Nutr 2009, 90:935-942

20. O'Connor TM, Yang SJ, Nicklas TA: Beverage intake among preschool children and its effect on weight status. Pediatrics 2006, 118:e1010-e1018.

21. (NCHS) National Center for Health Statistics: Overview: NHANES Sample Design. http://www.cdc.gov/nchs/tutorials/Nhanes/SurveyDesign/ SampleDesign/intro.htm (accessed December, 2010).

22. U.S. Department of Health and Human Services/ U.S. Department of Agriculture: Dietary Guidelines for Americans; 2005. [http://www.cnpp.usda. gov/DGAs2005Guidelines.htm http://www.health.gov/dietaryguidelines/ dga2005/report/HTML/D10_Conclusions.htm] (accessed December 2010).

23. Krebs-Smith SM, Guenther PM, Subar AF, Kirkpatrick SI, Dodd KW: Americans do not meet federal dietary recommendations. J Nutr 2010, 140(10):1832-1838.

24. Kaitosaari T, Ronnemaa T, Raitakari O, Talvia S, Kallio K, Volanen I, Leino A, Jokinen E, Välimäki I, Viikari J, Simell O: Effect of 7-year infancy-onset dietary intervention on serum lipoproteins and lipoprotein subclasses in healthy children in the prospective, randomized Special Turku Coronary Risk Factor Intervention Project for Children (STRIP) study. Circulation 2003, 108(6):672-677.

25. Kaitosaari T, Ronnemaa T, Viikari J, Raitakari O, Arffman M, Marniemi J, Kallio K, Pahkala K, Jokinen E, Simell O: Low-saturated fat dietary counseling starting in infancy improves insulin sensitivity in 9-year-old healthy children: the Special Turku Coronary Risk Factor Intervention Project for Children (STRIP) study. Diabetes Care 2006, 29(4):781-785.

26. Daniels SR, Greer FR, Committee on Nutrition: Lipid screening and cardiovascular health in childhood. Pediatrics 2008, 122:198-208.

27. Murphy MM, Douglass JS, Johnson RK, Spence LA: Drinking flavored or plain milk is positively associated with nutrient intake and is not associated with adverse effects on weight status in US children and adolescents. J Am Diet Assoc 2008, 108:631-639.

28. Gidding SS, Dennison BA, Birch LL, Daniels SR, Gillman MW, Lichtenstein AH, Rattay KT, Steinberger J, Stettler N, Van Horn L: American Heart Association; American Academy of Pediatrics: Dietary recommendations for children and adolescents: a guide for practitioners: consensus statement from the American Heart Association. Circulation 2005, 112:2061-2075.

29. Rampersaud GC, Bailey LB, Kauwell GP: National survey beverage consumption data for children and adolescents indicate the need to encourage a shift toward more nutritive beverages. J Am Diet Assoc 2003, 103:97-100

30. Harnack L, Stang J, Story M: Soft drink consumption among US children and adolescents: nutritional consequences. J Am Diet Assoc 1999, 99:436-441.

31. Fiorito LM, Marini M, Mitchell DC, Smiciklas-Wright H, Birch LL: Girls' early sweetened carbonated beverage intake predicts different patterns of beverage and nutrient intake across childhood and adolescence. J Am Diet Assoc 2010, 110:543-550.

32. Fisher JO, Mitchell DC, Smiciklas-Wright H, Mannino ML, Birch LL: Meeting calcium recommendations during middle childhood reflects motherdaughter beverage choices and predicts bone mineral status. Am J Clin Nutr 2004, 79:698-706

33. Guo SS, Chumlea WC: Tracking of body mass index in children in relation to overweight in adulthood. Am J Clin Nutr 1999, 70(1 Part 2):145S-148S.

34. Dennison BA, Rockwell HL, Baker SL: Excess fruit juice consumption by preschool-aged children is associated with short stature and obesity. Pediatrics 1997, 99:15-22

35. Alexy U, Sichert-Hellert W, Kersting M, Manz F, Schoch G: Fruit juice consumption and the prevalence of obesity and short stature in German preschool children: results of the DONALD study. Dortmund Nutritional and Antropometrical Longitudinally Designed. J Pediatr Gastroenterol Nutr 1999, 29:343-349.

36. Wiley AS: Dairy and milk consumption and child growth: Is BMI involved? An analysis of NHANES 1999-2004. Am J Hum Biol 2010, 22:517-525.
37. Huh SY, Rifas-Shiman SL, Rich-Edwards JW, Taveras EM, Gillman MW: Prospective association between milk intake and adiposity in preschoolaged children. J Am Diet Assoc 2010, 110:563-570.

38. Woteki CE: Measuring dietary patterns in surveys. Vital Health Stat 1992, 4:101-108

39. Beer-Borst S, Amadò R: Validation of a self-administered 24-hour recall questionnaire used in a large-scale dietary survey. Z Ernährungswiss 1995, 34:183-189.

doi:10.1186/1475-2891-11-92

Cite this article as: Fulgoni and Quann: National trends in beverage consumption in children from birth to 5 years: analysis of NHANES across three decades. Nutrition Journal 2012 11:92

\section{Submit your next manuscript to BioMed Central and take full advantage of:}

- Convenient online submission

- Thorough peer review

- No space constraints or color figure charges

- Immediate publication on acceptance

- Inclusion in PubMed, CAS, Scopus and Google Scholar

- Research which is freely available for redistribution 